\title{
Los repertorios léxicos de carácter autónomo de la medicina dieciochesca en lengua española
}

\section{Autonomous Lexical Repertoires in the 18th Century Medicine Written in Spanish}

Josefa Gómez de Enterría SÁnchez [j.gomezdeenterria@uah.es] Universidad de Alcalá, España

\section{RESUMEN}

Durante el siglo XVIII, la renovación de la ciencia favorece el nacimiento del lenguaje científico moderno, con el consecuente desarrollo de numerosos vocabularios de especialidad que, paralelos a la evolución del saber, verán la luz a lo largo del siglo ilustrado. Es entonces cuando afloran los primeros repertorios de especialidad que, a lo largo del siglo XIX afianzarán la lexicografía especializada en lengua española con su progresivo desarrollo, constituyéndose en cauce imprescindible para la difusión de las nuevas terminologías. Este trabajo pone el foco en los diccionarios que en el siglo XVIII prestan especial atención al nuevo léxico de la medicina.

\section{Palabras clave}

Siglo XVIII; neologismos de la medicina en español; repertorios de especialidad; traducción especializada

\begin{abstract}
During the 18th century Science renovation favors the birth of modern scientific language; as a consequence, many specialized vocabularies will appear throughout the Enlightenment Century, in parallel with the evolution of knowledge. In this period the first specialized repertoires are published, and they will strengthen specialized lexicography in Spanish, as they become an essential way of spreading new terminologies. This paper analyses some 18th century dictionaries that pay special attention to the new medicine lexicon.
\end{abstract}

\section{KEYWORDS:}

18th century; medicine neologisms in Spanish; specialised repertoires; specialised traduction

RECIBIDO 2020-05-10; ACEPTADO 2020-08-30 


\section{Introducción}

Durante el siglo XVIII se produce la consolidación de la ciencia y la creación de nuevas áreas científicas independientes. Es entonces cuando las lenguas vernáculas alcanzan plenamente el estatus de lenguas nacionales para la difusión del conocimiento, erigiéndose en cauce prioritario para la comunicación especializada y relegando a reductos limitados las publicaciones científicas escritas en latín. El empleo de las lenguas vernáculas será el detonante que facilite el proceso de popularización de la ciencia en toda Europa; así lo evidencia la elevada producción editorial de tratados científicos en las lenguas de cultura europeas y, más aún si cabe, en la nuestra, donde ya desde el Renacimiento existía, en la medicina, una literatura científica en romance (Gómez de Enterría 2020: 63).

En España, la renovación de la ciencia va a favorecer el nacimiento de un lenguaje científico moderno, con el consecuente desarrollo de numerosos vocabularios de especialidad que, paralelos a la evolución del saber, verán la luz a lo largo del siglo ilustrado. Al mismo tiempo, también afloran los primeros repertorios de la lexicografía especializada en lengua española, bien los diccionarios de especialidad de carácter autónomo o bien los glosarios escondidos, estos últimos como elementos auxiliares de lectura de los tratados y manuales (Álvarez de Miranda 2011: 168).

\section{El interés por los repertorios de especialidad en el s. XVIII}

El afianzamiento de la ciencia en Europa y, en consecuencia, la creación de nuevas áreas científicas favorecerá, desde finales del siglo XVI, la publicación de numerosos diccionarios de especialidad. El ejemplo más patente es quizá el que nos proporcionan los repertorios geográficos e históricos, estos "se convirtieron pronto en un instrumento como arsenal ordenado de noticias y como medio para detectar las contradicciones de las fuentes antiguas y medievales" (Capel 1981), tal como exigía el desarrollo de una historia crítica sustentada en fechas y lugares. Estos repertorios aplicarán el orden alfabético propio de los diccionarios de lengua, que ya poseían una larga tradición y de los que el ejemplo pionero fue el diccionario de Nebrija, publicado a comienzos del siglo XVI.

Desde las últimas décadas del siglo XVII, y a consecuencia de la revolución científica de los novatores, se había despertado en Europa gran interés por dominar el conocimiento en toda su dimensión ${ }^{1}$. Los novatores tratarán de hacer acopio de la mayor extensión posible de los saberes del momento para reunirla en repertorios; lo más interesante de esta actitud es que derivará en el enciclopedismo ilustrado, una vez avanzada la mitad del siglo. En Francia, el antecedente más inmediato de este nuevo fenómeno es el Dictionnaire historique et critique de Pierre Bayle (1697), aunque conviene recordar que ya existían obras que podemos considerar de carácter enciclopédico desde la antigüedad, como los llamados speculum, thesaurus o summa. Pero va a ser en la centuria ilustrada cuando el afán del saber despierte en los filósofos franceses la necesidad de

1 Esta disposición también se aprecia en algunos repertorios como, por ejemplo, en la traducción del francés al español del Universo abreviado a donde están contenidas en diversas listas casi todos los nombres de las obras de la naturaleza de todas las ciencias y de todas las artes de François Antoine Pomey, traducida por el jesuita Croiset, y publicada en Lyon en 1705 . 
compendiar todos los saberes, no solo los científicos sino también los de carácter técnico, desembocando en la creación del más importante proyecto enciclopédico de nuestra historia cultural: la Encyclopédie francesa. Esta magna obra nace inspirándose directamente en la Cyclopaedia de Ephraim Chambers (Londres, 1228), que había alcanzado gran éxito en Inglaterra, extendiendo su influencia rápidamente por Europa hasta el punto de que, en un corto periodo de tiempo, fue traducida a varios idiomas.

La Encyclopédie se concibe como un repertorio capaz de acoger la síntesis del conocimiento de toda una época a la luz de la razón y, siguiendo el modelo de la Cyclopaedia, se va a estructurar de acuerdo con el orden alfabético, aunque con un valor añadido, pues favorece también la organización temática mediante ontologías que organizan el conocimiento (Cordón García 1996: 121). En la redacción de la Encyclopédie interviene un nutrido grupo de especialistas que la redactarán bajo la dirección de Diderot (1713-1784) y D’Alembert (1717-1783), en los años comprendidos entre 1751 y 1775; es en este lapso de tiempo cuando irán viendo la luz los sucesivos volúmenes hasta completar el número de diez. Uno de sus logros más importantes consiste en que es precisamente la Encyclopédie la obra que va a fijar las características de la enciclopedia moderna.

\section{La importancia de la traducción para la creación de los vocabularios de especialidad}

En el origen de la popularización de la ciencia en la Europa dieciochesca es preciso considerar la intensa actividad traductológica, que dio como resultado el elevado número de obras científicas vertidas a las lenguas vernáculas. Pero, simultáneamente al fenómeno traductor, también comienzan a despuntar los primeros diccionarios de especialidad, propios de cada ámbito del saber, que, además de facilitar el desarrollo de la divulgación científica durante la centuria ilustrada, tuvieron capital importancia para el nacimiento de los nuevos vocabularios de especialidad.

En España, la actividad traductora se incrementa de forma considerable a lo largo del siglo XVIII con la traducción de numerosas obras científicas, sobre todo durante la segunda mitad, con lo que esto representó para el desarrollo del país en los ámbitos científico, económico y cultural. Estas traducciones poseían características propias, pues fueron realizadas por un colectivo que tenía varios rasgos en común ${ }^{2}$, de ahí que generalmente fundamentaran sus asertos teóricos en torno al concepto de mimesis, valorando la verdad por encima de cualquier otra consideración, de manera semejante a la que emplea el historiador al abordar su tarea (West 1932: 346).

El interés que despiertan las traducciones científicas dieciochescas, para el estudio de los diccionarios de especialidad, dimana del tratamiento que reciben los neologismos en las versiones, porque son las traducciones de la ciencia las que aportan las nuevas ideas y los nuevos conceptos con la consiguiente introducción de las nuevas voces y, por ende, la creación de las nuevas terminologías ${ }^{3}$. Es importante señalar que la mayoría de las veces los traductores de los textos científicos

2 No es anecdótico el hecho de que la mayoría de las versiones realizadas en esta etapa nos haya permitido corroborar que fueron realizadas por verdaderos especialistas en la materia traducida (Gómez de Enterría 2020: 85).

3 La nueva lengua de la botánica nos ofrece un caso singular en el siglo XVIII. Esta lengua de especialidad ya estaba implantada en Francia desde los comienzos del nuevo siglo, donde había sido desarrollada por Tournefort con la clasificación de carácter descriptivo del reino vegetal, a partir de las características de flores y plantas. Sin embargo, la 
adoptan ante el neologismo una postura favorable y son conscientes de la importancia que tienen las nuevas nomenclaturas para el desarrollo de la ciencia; así lo hacen ostensible muchas veces en los prólogos de sus versiones o en numerosas notas al pie . $^{4}$

No es de extrañar que la corriente purista, tan activa en la lengua literaria a lo largo de todo el siglo XVIII, apenas tuviera eco en los textos de la historia natural ni en los de la medicina vertidos al español a lo largo de la centuria, a pesar de que en el primer tercio del siglo harán su aparición algunas tendencias de defensa de la lengua científica, que irrumpen a la par del desarrollo de las sucesivas escuelas. Sirvan como ejemplo, en la medicina, los enfrentamientos entre latinistas y romancistas durante las primeras décadas del siglo ilustrado (Gómez de Enterría 2010: 57-59), y en la botánica el desacuerdo que resulta entre las dos corrientes nomencladoras, cuando tratadistas y traductores toman partido por la nomenclatura linneana frente a la diseñada por Tournefort, que además no dudan en afirmar rotundamente la importancia que la nueva terminología tiene para el desarrollo de la ciencia. No ocurre así en otros círculos del saber ilustrado como, por ejemplo, entre los traductores de obras de economía, cuando evitan el empleo de las nuevas voces sólo porque las consideran ajenas a la lengua, además del problema añadido que en su momento representa, para cualquier traducción, la censura previa a su aprobación y el rechazo que muchas veces producen esas mismas voces en algunos censores ${ }^{5}$.

solución taxonómica de la terminología botánica llegará a Europa algunas décadas más tarde, con la capital aportación de la nueva clasificación sistemática del naturalista sueco Linneo (1736: 63), que será adoptada como cauce de comunicación por todos los científicos del mundo.

4 Esta actitud se evidencia en el proemio de la traducción al español de Elementos de la nomenclatura botánica y sistema sexual de las plantas, realizada en 1802 por el médico y botánico ilustrado Juan Francisco Bahí, cuando escribe: "[...] y habiendo yo visto con satisfacción los elementos del Doctor Plenk más ricos y completos en las nociones de la nomenclatura botánica, para los que se dediquen a esta tan importante y deleitosa ciencia, creí hacer algún servicio a estos, sobre todo a los discípulos de los Reales Colegios de Cirugía-Médica, para quienes en particular he sacrificado este pequeño trabajo, si les daba en lengua vulgar la traducción de aquellos"; y sigue más adelante argumentando a favor de las nuevas terminologías con el ejemplo de otra de las más novedosas y de más brillante sistematización: "Una gran parte de los adelantamientos que han hecho en estos tiempos las ciencias naturales se debe a su nueva y arreglada nomenclatura: la Chímica lo ha comprobado bien, cuyas voces, dando a entender muy de pronto lo que se busca o se trata, facilitan la adquisición de la ciencia, la hacen más común, y así entre muchos más que la cultivan se proporcionan cada día nuevos encuentros" (Bahí [Plenck] 1802: 22). Si bien es cierto que también el autor de la obra traducida, Joseph Jacobo Plenck (1735-1807), prestigioso médico y botánico, expone en el cuerpo de la obra su preocupación acerca de la importancia que posee la nomenclatura para el desarrollo de la ciencia y así, al plantear el fundamento de la ciencia, afirma: "El primer fundamento de la botánica es la terminología o nomenclatura botánica, la cual enseña el describir bien y distintamente cada parte de la planta. Aquella sola facilita y hace cierto el conocimiento de los vegetales" (Bahi [Plenck] 1802: 31). Esta actitud del científico, con verdadera vocación de terminólogo, se constata, pues, en las obras de Joseph Jacobo Plenck con el enriquecimiento léxico que este médico austriaco aportó al vocabulario de la medicina con numerosos neologismos, sobre todo en sus tratados de medicina y farmacología (Gómez de Enterría 2020). Un ejemplo nos lo proporciona cuando introduce la nueva voz higroma en su Tratado de las enfermedades de los ojos: "Opthalmoptosis por tumor dentro de la órbita. Un exostose, tofo, absceso, lipoma, atheroma, hygroma, escirro, nacidos dentro de la órbita, o la gordura de la misma órbita endurecida, exprimen o empujan el globo del ojo arriba, abaxo o acia los ángulos" (Vidal [Plenck] 1797: 92). Este neologismo fue recogido por primera vez en el siglo XIX en el Diccionario Nacional de Ramón Joaquín Domínguez (1853): "higroma, s. m. Patol. Quisto (sic) acuoso, aislado y lleno de linfa, del tamaño de un huevo".

5 Los censores algunas veces llegaban incluso a considerar que el neologismo podía ser uno de los cauces para la introducción de las "nuevas ideas contrarias a la santa religión y a las regalías de Su Majestad” (Gómez de Enterría 1996). 


\section{Los repertorios de especialidad del s. XVIII en español, con especial atención a los vocabularios de medicina}

Como ya hemos visto, la renovación de la ciencia favorece el nacimiento de las nuevas terminologías, con el consecuente desarrollo de los vocabularios de especialidad. Estos verán la luz organizados en repertorios léxicos, además de los llamados glosarios escondidos, tan frecuentes desde el siglo XVI en algunos ámbitos del conocimiento como la medicina y la botánica. Estos glosarios se incluyen en el interior de las obras con una finalidad didáctica, su objetivo era proporcionar el esclarecimiento de las voces a los posibles lectores, de ahí que lleguen a alcanzar gran valor. Su importancia reside en que, además de su utilidad, gracias a la novedad de algunos de sus términos, aportan también un interés añadido a la obra que los contiene (Gutiérrez Rodilla 2010: 65). Entre los más sobresalientes está el que incluye el médico Andrés Laguna en su versión castellana de Materia médica de Dioscórides, publicada en 1555. También en este mismo siglo, el glosario que el médico sevillano Hidalgo de Agüero incluye en su Thesoro de la verdadera Cirugía y vía particular contra la común ${ }^{6}$ (1604). Por las mismas fechas y en el ámbito de la botánica destaca el que publica el fraile mercedario Miguel Agustín con el título "Vocabulario de seis lenguas, en que se declaran los nombres de los árboles, yervas, frutas, y otras cosas, contenidas en el presente Libro de los Secretos de Agricultura". Se trata de otro de los denominados glosarios escondidos, pues ocupa unas veinte páginas del Libro de los secretos de agricultura, casa de campo, y pastoril, traducido de lengua catalana al castellano por Fray Miguel Agustín ${ }^{7}$ a partir del original que él mismo había publicado anteriormente en catalán. Pero será en la edición de 1626 cuando, al hacer la versión castellana, el prior le añadirá un tomo más, incluyendo también el vocabulario en seis lenguas. El éxito de la obra fue enorme, hasta el punto de que se reeditó reiteradamente a lo largo del siglo XVIII.

El objetivo principal de los repertorios de especialidad fue inventariar y ordenar las voces propias del ámbito de especialidad propuesto ${ }^{8}$ para describirlo y poder acercarlo hasta el lector, ofreciéndolo mediante un vocabulario organizado en orden alfabético, a la manera ya tradicional de los diccionarios de lengua. De donde podemos afirmar que el siglo XVIII favoreció el nacimiento de la lexicografía especializada, abarcando no solo las áreas del conocimiento que venían desarrollándose desde siglos anteriores como la botánica, la historia natural o la medicina, sino también otras ramas del saber que eran totalmente nuevas en la centuria ilustrada o estaban aún poco desarrolladas, como por ejemplo el comercio o la economía?

La medicina nos ofrece el panorama de una ciencia que posee un vocabulario fuertemente afianzado desde la antigüedad clásica, con algunos períodos en lengua vernácula muy brillantes como fue el Renacimiento en España, cuando se desarrolla un léxico médico en romance castellano de enorme riqueza. Las primeras décadas del siglo XVIII transcurren entre las discusiones

6 Se trata de una obra recopilatoria de varios escritos que fue publicada después de la muerte del autor.

7 Miguel Agustín (1560-1630) fue prior de la iglesia de San Juan de Perpignan.

8 En ocasiones los inventarios también consideraban la necesidad de recoger además las voces relativamente nuevas, contribuyendo así a la sistematización de la ciencia.

9 Véase el interesante trabajo de Astigarraga y Zabalza (2006) sobre el interés de numerosos ilustrados para elaborar diversos diccionarios de economía y comercio en España desde comienzos del siglo XVIII, obteniendo algunos logros interesantes con la publicación en las últimas décadas de repertorios como los de V. Alcalá-D. Gallard, M. G. Suárez, y T. A. Marien. 
doctrinarias de los médicos iatromecánicos, partidarios de la patología mecanicista, y los iatroquímicos, comprometidos con la fisiología química, situando la ciencia médica una vez más en la querella entre antiguos y modernos. Es entonces cuando el empirismo racionalizado va a reaccionar frente al galenismo, aún imperante en la medicina tradicional española de los años centrales de la centuria. Este contexto de fuertes polémicas científicas y enfrentamientos disciplinares resultará muy propicio para la creación de interesantes repertorios que acogen los vocabularios de especialidad de la medicina y áreas afines. En este período es muy frecuente la presencia de glosarios escondidos en los manuales y tratados, de entre los que podemos citar, a manera de ejemplo, los siguientes: el que incluye Juan Bautista Juanini en su obra Nueva idea física, de 1685; Manuel de Porras en la Anatomía galénica moderna, de 1716; Monravá y Roca en su Breve curso de nueva cirugía, de1728; y Martín Martínez en su Examen de cirugía moderna, de 1725, y en la Anatomía completa del hombre, de 1728. Este médico novator, junto con sus obras, es una de las referencias más citadas en el Diccionario de Autoridades y en el Diccionario Castellano de Esteban de Terreros para autorizar las voces de la medicina.

Entre los repertorios autónomos citaremos los tres más destacados durante siglo XVIII, los diccionarios de Ruyzes de Fontecha, Suárez de Ribera y Terreros.

\subsection{El Diccionario de Juan Alonso y de los Ruyzes de Fontecha}

Juan Alonso y de los Ruyzes de Fontecha ${ }^{10}$ publica en fecha temprana, a comienzos del siglo XVII, un "Diccionario de los nombres de piedras, plantas, fructos, yervas, flores, enfermedades, causas y accidentes" que incluye en el tratado Diez previlegios para mujeres preñadas, que vio la luz en 1606. Este Diccionario, organizado alfabéticamente, acoge entradas de botánica, patología, anatomía, farmacopea y mineralogía. Según expone Zabía Lasala (1999: IX-XXIII) en la excelente edición que hace a partir del texto original, uno de los objetivos fundamentales de Alonso de los Ruyzes fue clarificar el panorama terminológico del léxico de la botánica y la medicina, sin olvidar las otras áreas afines, en un momento histórico de superposición léxica y de fuerte vacilación. Esta era la situación en que se encontraba la medicina de los siglos áureos, fuertemente influenciada por el legado antiguo grecolatino, además de la medicina medieval latina y la ciencia islámica. La lengua de la medicina en el siglo XVII era un crisol, en el que convivían, de una parte, la tradición galénica recibida a través de las traducciones latinas y arábigas, sin olvidar el fuerte arraigo que aún tenía en la península el vocabulario de la medicina árabe; y, de otra parte, la pervivencia de la tradición romancista del Renacimiento en la medicina hispana.

En este contexto, el diccionario de Ruyzes trata de dar una visión más o menos uniforme del variado y complejo mundo de la sinonimia de las voces especializadas que aún perduraban desde el siglo XV (como, por ejemplo: "alchalchum, la nuez, cabeça y principio del áspera arteria", "alhach, la cabeça de la gula, tragadero", "abhear, dispnea, dificultad de respirar, especie de asma", "abietina, terebentina, trementina del abeto", “alsacheichalnema, las hamapolas rubias, papauerruber”, etc.), proponiendo la voz que él considera más precisa, pero dejando además constancia de las voces que, con

10 Estudió medicina en la Universidad de Alcalá de Henares, donde se licenció en 1587; allí fue también catedrático de Vísperas. En 1605 ocupó una cátedra en Parma, Italia. Perteneció a la escuela de Alcalá. (Zabía Lasala 1999: X). 
distintas acepciones, se prestaban a confusión. Es así como Ruyzes va a facilitar el paso previo para la acuñación de numerosos préstamos semánticos, tomados de la medicina hipocrática y galénica como en: "cephalalgia, dolor de cabeça de poco tiempo, o cefalargia o kefalargia" "cubitus, el cobdo, parte del braço", "districhiasis, mal de ojos por demassiados pelos", etc. De la misma manera que observamos en los glosarios escondidos del Renacimiento, este arduo trabajo lexicológico, realizado por Juan Alonso, tenía una clara finalidad didáctica, pues se proponía acopiar en un diccionario todo el vocabulario que el estudiante de la época pudiera necesitar al leer los libros de medicina.

Desde el punto de vista lexicográfico, el Diccionario de Fontecha ofrece algunas características que lo hacen singular. En primer lugar, hay que tener en cuenta que no estamos ante un diccionario del español de la medicina y la botánica, porque su autor concibe el diccionario desde el latín y es esta la lengua de gran parte de los lemas, además de los transliterados del latín o del griego o de ambos, con el resultado de términos híbridos romanceados, con escasas entradas en lengua romance. Sin embargo, al redactar cada artículo el autor proporciona numerosos sinónimos en romance, así toma cada uno de los lemas que forman la nomenclatura del diccionario desde las traducciones latinas, vertidas con frecuencia de las fuentes árabes y griegas, de ahí que podamos encontrar muchas voces que son transliteraciones o también términos híbridos transliterados (Zabía Lasala 1999: XII). Otro rasgo peculiar es que Fontecha considera las diferentes variantes gráficas de un mismo término como entradas, sin respetar fielmente el orden alfabético. Sus definiciones son por lo general muy breves y repiten muchas de ellas la misma estructura. Estamos, pues, ante un valioso repertorio para el estudio de la historia del léxico de especialidad de la botánica, la medicina y otras áreas afines.

\subsection{El Diccionario Medico de Suárez de Ribera}

Francisco Suarez de Ribera (1686 - 1738) ${ }^{11}$ se propone escribir el primer diccionario de historia natural en lengua española, que publica en 1730-1731 con el título Clave Medico-Chirurgica y Diccionario Medico. Este ambicioso Diccionario terminológico de contenido anatómico, mineralógico, botánico, zoológico, farmacéutico, químico e histórico-físico, con especial atención a la medicina y un claro predominio de la botánica ${ }^{12}$ sobre las demás áreas, enfatiza el interés que Suárez de Ribera siente por los vocabularios de especialidad, pues es consciente de la importancia que el conocimiento representa para el especialista (médico, botánico, etc.) ${ }^{13}$, así lo

11 Estudió en la Universidad de Salamanca, donde se licenció en medicina y también obtuvo allí el doctorado. Durante una larga estancia en Sevilla, que determinó su orientación en la profesión médica, llegó a ingresar como miembro de la Regia Sociedad Médica de esta ciudad, situándose en un ámbito difuso que mediaba entre la tradición galénica, la iatroquímica y el empirismo clínico; esta postura lo llevó a polemizar muy frecuentemente con prestigiosos ilustrados como el Dr. Martín Martínez y el Padre Feijoo, entre otros. Posteriormente se estableció definitivamente en la corte, donde en 1733 obtuvo el nombramiento de médico de Cámara. Fue un prolífico autor de obras de medicina, publicadas a partir de 1718.

12 La iconografía que acompaña a los artículos de botánica son interesantes ilustraciones realizadas con gran precisión y rigor, se trata de grabados en madera y aguafuertes de gran valor.

13 El Proemio de Clave Medico-Chirurgica y Diccionario Medico atestigua esta preocupación del autor cuando escribe: "Aquel que quiere saber, y entender, debe primero tener perfecta inteligencia de los nombres, que se usan en la Ciencia Médica, pues son muchos, y varios, [...] quien no sabe la discrepancia de voces, que hay entre Antiguos, y Modernos cree que son diversas cosas, siendo unas mismas las substancias, aunque diversas las voces” (Suárez de Ribera 1730-I: 2). 
hace patente en su extensa producción editorial, con una presencia siempre constante de neo$\operatorname{logismos}{ }^{14}$.

Este diccionario nace con una decidida vocación comunicadora y en consecuencia presto para la divulgación, como pone de manifiesto el nutrido acopio de contenidos que Suárez de Ribera reúne en el cuerpo de la obra; claro testimonio del compromiso que este médico había adquirido con la terapéutica, como podemos comprobar en sus numerosos escritos, de entre los que destacan los dedicados a comentar y recomendar remedios secretos ${ }^{15}$. Estamos ante una obra muy ambiciosa que a todas luces adolece de un plan previo, tal vez esta haya sido la causa principal por la que tan interesante proyecto se truncó en la letra C, cuando apenas estaba comenzando. La Clave Medico-Chirurgica y Diccionario Medico es un repertorio sin criterio lexicográfico, como muestran la falta de unificación y la considerable desproporción de la obra en todos sus aspectos. Esta desproporción salta a la vista cuando, en una primera ojeada, observamos el tratamiento verdaderamente dispar que reciben sus artículos. Así, son numerosas las entradas de escueta definición, muy concisa y con frecuencia acompañada de la equivalencia que corresponde al lema en lengua vulgar: "acoros, es una hierba llamada espadaña silvestre”, "arzolla, este nombre castellano significa a la almendra verde". Sin embargo, junto a esta clase de artículos, también acoge otros con definiciones enciclopédicas, en las que la descripción del concepto puede abarcar una o dos páginas, como leemos por ejemplo en la entrada acetum. Aunque el enciclopedismo de Ribera no se limita a ejemplos como el anterior, sino que puede llegar hasta la desproporción total, como podemos comprobar en el artículo acidum sanguinis, donde el autor, después de definir la voz de manera concisa ("acidum sanguinis, es el ácido que en estado natural se halla, en la sangre”), lo amplía hasta abarcar treinta y siete páginas en las que describe, con todo género de detalles, la importancia de este concepto desde la antigüedad hasta el siglo XVIII, exponiendo los planteamientos teóricos y dando testimonio de las sucesivas y numerosísimas controversias entre partidarios y detractores del mismo, a los que denomina acidistas y antiacidistas respectivamente ${ }^{16}$.

La misma ausencia de criterio lexicográfico está presente en la manera que tiene Ribera de ofrecer las equivalencias de las voces en numerosas lenguas: alemán, francés, inglés, italiano, por-

14 Sus publicaciones nos proporcionan muy a menudo primeras dataciones de neologismos como: antiemético, antiescorbútico, antiescrofuloso y antihistérico, datadas en 1721; antiepiléptico, antihelmíntico y antiparalítico, en 1732; antiespasmódico, en 1739; botiquín, clorosis y clorótico, en 1730 (Gómez de Enterría 2020).

15 No es de extrañar, pues, que Ribera fuera el introductor en España de las obras más exitosas sobre remedios curativos, que habían adquirido gran predicamento en la Europa del momento. Las más representativas fueron la Pharmacopoea Bateana del inglés William Bate, que Ribera difundió entre los boticarios, y las obras del médico portugués João Curvo Semedo (1635-1719). Suárez de Ribera tradujo al español las obras más importantes de este médico portugués, cuya larga vida estuvo dedicada a la preparación de medicinas y a la clínica. Así lo expone en el prólogo de Observaciones de Curvo compendiadas e ilustradas por Suárez de Ribera, 1735 [1733]: “[...] tomé a mi cargo el traducirlas del Idioma portugués al castellano, de compendiarlas, e ilustrarlas con admirables arcanos medicinales, los que espero sirvan de mucha utilidad a la salud pública, pues son de no inferior gerarquia, que los arcanos de Lázaro Ribeiro, y de otros autores" (Suárez de Ribera [Curvo Semedo] 1733: Prólogo, s/p).

16 Son quizá estos desmanes lexicográficos los que indujeron a Colmeiro para juzgar a Ribera con gran dureza, como muestra al afirmar en su Botánica: "Esta obra, cuyo título manifiesta desmesuradas pretensiones en el autor, no se llegó a terminar, ni se perdió mucho en ello [...]. Está sembrada de extravagancias, como todos los escritos del mismo autor" (Colmeiro 1858: 70). Este ilustre médico y botánico del siglo XIX únicamente salva de entre las publicaciones de Ribera el Dioscórides, cuando escribe: "La mejor publicación que se debe a Suárez de Ribera es la del Pedacio Dioscorides, anotado por Laguna, nuevamente ilustrado y añadido, que se imprimió en Madrid, como las demás obras, en el año 1733" (Colmeiro 1858: 160). 
tugués, árabe, hebreo, griego, etc., porque no solo lo hace aleatoriamente, es decir, sin ningún criterio previo, sino que muchas veces incluye el lema directamente en la lengua extranjera, así podemos leer: "abobora, es un término portugués que significa a la calabaza. Vide cucúrbita", "ache, es voz francesa, que significa a la hierba llamada apio", "adaquamento, es voz con que en italiano se apellida la irrigación", "acciayo, es voz con la que en italiano se nombra al hazero", "adlah, es nombre, que en arábigo significa al músculo", “adamsapefel, es voz que en alemán significa aquella parte de la garganta, llamada manzana de Adam, o nuez del tragadero", "adlerstein, es voz con que en lengua germánica, o alemana se nombra a la piedra del águila", "anckle, es término inglés con que se nombra el hueso del pie, llamado maléolo", "anat, es dicción inglesa con que se apellida una planta. Vide anethum", "bakle, es voz con que en hebreo se nombra la verdolaga", "baktanden, en bélgico significa los dientes", "baladre, es nombre que en catalán significa la delfa”. Tal vez esta proliferación de lemas en idiomas tan diversos sea lo que le lleva a redactar otros artículos en los que aclara que se trata de palabras de la propia lengua, como los siguientes: "babosa, es nombre castellano. Vide babaza". Y al buscar esta: "babaza", en castellano es voz con que se nombra a un gusano a manera de sanguijuela que habita comúnmente en lugares húmedos. Vide limax”. Pero no termina aquí la confusión, porque Ribera otras veces incorpora, dentro del cuerpo de la obra, otras voces de las que no da filiación alguna, a pesar de que no se trata de palabras castellanas; en estas últimas lo más que hace es proporcionar algunas veces cierta orientación sobre la marca técnica: "adibat, según los chímicos, significa al mercurio", "admisurab, es un término, que significa a la tierra", "adehemest, es voz que significa lo mismo que lamina".

También incluye Ribera en su Clave Medica algunas voces de la Nueva España, aunque escasas y con un tratamiento bastante irregular, semejante al del resto de las entradas. Así leemos: "acoati, es una serpiente aquatil, llamada por otro nombre miocoatl, cuyo color imita a la espiga del maíz, sus dientes pequeños, y se cría en las lagunas, y aguas detenidas de las regiones templadas, y su mordedura no tiene peligro". Se refiere Ribera al indigenismo acuate ${ }^{18}$, del que no ofrece marca alguna sobre su ámbito de uso. Otras veces, sin embargo, nos da la información del contexto geográfico mediante procedimientos diversos como en "acameti, según los mexicanos, es el azíbar", o "brionia mexicana, assí se nombra la Jalapa".

Es frecuente que en algunas entradas proporcione las formas variantes de la voz, ya sea dentro del cuerpo del artículo (como en "abricoc, es un término francés, que significa aquel fruto que en castellano llamamos alvarcoques y albérchigos", "abesamum, es el lodo de que usan los chímicos, y le llaman lodo de Rota") o acompañando al propio lema, como en: "adarces, adarce, adarción, y adarcus, son voces sinónomas (sic), que significa (sic) aquella substancia salsuginosa, coagulada en las cañas, y hierbas marítimas, en quienes azota el agua salada; y esta substancia es la que

17 Esta definición está tomada directamente -con muy ligeras variantes- de la del Diccionario de Autoridades (1726): "Babaza. Cierto gusano de color obscuro de la hechura de una sanguijuela, algo menor en el tamaño, el qual se cría ordinariamente en los lugares húmedos. Llámase Babaza porque en pisándole, u deshaciéndole se convierte todo en babas. Llámase también Babosa. Lat. Limax".

18 Agradezco a mi colega y buen amigo, el profesor Ramírez Luengo, la información que me ha proporcionado sobre la voz acoati que trae Ribera en su Clave Medica. Se trata de un indigenismo formado con la base coatl 'serpiente', cuya adaptación al español se hace con /e/, que junto con la diptongación del hiato da la forma 'acuate'. En el Diccionario de Americanismos de Santamaría: "Acuate: (Del azt. ail, agua; coatl, culebra.) m. Culebra de agua de unos $50 \mathrm{cmts}$. de largo; dientes muy chicos, cabeza negra por encima, azul a los lados, y amarilla abajo; la espalda listada de negro y azul y la barriga toda azul" (Santamaría 1942: s.v. acuate). 
algunos llaman espuma maris, y con esta voz la suelen pedir en algunas recetas de medicamentos exteriores, que para resolver eficazmente se aplican". Este último artículo nos proporciona además una muestra del discurso farragoso del que hace gala el autor en la mayoría de las definiciones descriptivas.

Ahora bien, no podemos pasar por alto que Ribera acoge en su repertorio numerosas voces neológicas para las que proporciona la primera datación lexicográfica, sobre todo las relacionadas con remedios y curaciones, como: antifebrile, antihectica, antihelmintica, antinephritica, antiparalitica, antipthisica, antipodagrica, antiscorbutica, antidysenterica, anticolica, antidiabetica, antystherica, antiartritica, antipleuriticum, antipireticum, antispasmodicum, etc., conservando estas la forma latina de los tratados de donde las había tomado, es decir, como extranjerismos en proceso de adaptación.

A la vista de tan insubsistente empresa, no es raro que Suárez Ribera no consiguiera culminar tan ambicioso proyecto, como el que muestran los tres volúmenes publicados, porque la ausencia de un criterio lexicográfico unificado lo inhabilita totalmente; el caos se hace ostensible como factor predominante en todo el diccionario, a pesar de sus notables dotes de divulgador y del extenso material recopilado.

\subsection{El Diccionario Castellano de Esteban de Terreros}

El tercer repertorio que nos proponemos comentar no es, como los anteriores, un diccionario de especialidad, sino que se trata de un proyecto mucho más abarcador. Su autor, el jesuita Esteban de Terreros y Pando, redacta el Diccionario castellano con las voces de ciencias y artes ([1767] 1786-1793), es decir, un repertorio que además de la lengua común acoge todas las voces de artes y ciencias que estaban en la lengua en el momento en que lo escribe. Pero al considerar la génesis del Diccionario es preciso recordar la faceta de Terreros traductor, porque el jesuita había vertido al castellano en fecha temprana la obra de Nöel Antoine Pluche, Le spectacle de la nature, ou entretiens sur les particularités de l'histoire naturelle, qui ont paru les plus propes à rendre les jeunes-gens curieux, et á leur former l'esprit, publicada en Paris en 1732. La traducción de esta obra enciclopédica verá la luz en Madrid entre los años 1753 y 1755, gracias a la diligencia y prontitud con que Terreros acometió tan magno proyecto, dado el escaso período de tiempo transcurrido entre la salida de las prensas de los ocho volúmenes del original francés y la finalización de la versión española, porque ya el 3 de agosto de 1750 Terreros solicitaba la licencia de impresión ${ }^{19}$. Estamos, pues, ante una traducción de ostensible singularidad, gran volumen y no menor importancia, gracias a la cual Esteban de Terreros se iniciaría en las tareas lexicográficas, como recuerda en el prólogo de su diccionario:

La primera determinación fue, habiendo traducido el Espectáculo de la Naturaleza, formar una especie de índice o vocabulario con las voces que busqué con sumo cuidado en las artes y en la naturaleza para este efecto, porque teniendo allí uno como tesoro de voces no se quedasen como sepultadas en solo aquella obra. Trabajando pues en esto y descubriendo con este cuidado y trabajo 
nuevos términos, me hicieron tomar la segunda determinación y fue la de formar un Diccionario de artes y ciencias, obra que concebía utilísima a la nación: con esta idea ya más ampla (sic) proseguía mi trabajo, cuando él mismo me advertía la suma dificultad de excluir de este Diccionario muchas de las voces comunes, y que aunque no las suelen traer los Diccionarios de artes y ciencias, con dificultad se pueden con razón separar de ellos. (Terreros y Pando 1767: XI).

Esteban de Terreros es consciente de que emprende un gran proyecto traductológico y lo aborda en profundidad aplicando grandes dosis de sentido común, pues ante la ausencia de repertorios sobre los temas de artes y ciencias presentes en los ocho volúmenes de la obra enciclopédica de Pluche - unas veces por falta de fuentes lexicográficas sobre la materia, otras por el caos terminológico que le proporcionaban los diccionarios de consulta- decide acopiar toda la documentación posible sobre cada una de las materias del Espectáculo y, cuando esto no fuera posible, apela a la consulta directa con los especialistas, como refiere en el prólogo:

Para este efecto ya no me armé tanto de libros, cuanto de constancia [...]. Todo el día estaba preguntando en la huerta, en el campo, en la tahona, en las tiendas, en las casas y en las calles. [...] Pasarán de quinientas personas las que han cooperado poco o mucho a estos informes [...] y solo el ser tantos puede hacer que no los nombre aquí mi reconocimiento, pues por su medio, y a expensas de su instrucción, me he hecho con un tesoro de términos facultativos, propios de las ciencias y artes. (Terreros y Pando [Pluche] 1753-1755: Prólogo del traductor, s/p).

En consecuencia, podemos afirmar que el corpus textual de partida ${ }^{20}$ para la elaboración del Diccionario fueron los 16 tomos del Espectáculo de la Naturaleza de Pluche, que Terreros había traducido al castellano con la idea de divulgar la ciencia; un verdadero acto de servicio para la nación española, acorde con los principios de la Ilustración, porque muestra en todo momento su conocimiento y amor por la lengua propia, valorándola y defendiéndola. También es importante señalar aquí que la actitud del Padre Terreros ante el neologismo será de aceptación plena, consciente de que las nuevas voces suponen un enriquecimiento para la lengua y que, al mismo tiempo, son el cauce para la renovación de ciencia y de la técnica. No es por ello de extrañar que su Diccionario acoja el conjunto léxico más rico y completo de todo el siglo ilustrado. Supera al primer diccionario académico en cerca de veinte mil voces y ofrece unos planteamientos metodológicos acordes con la exposición que Terreros da en el prólogo, verdadero tratado de lexicografía; así lo evidencia en cada uno de los artículos con definiciones, ya sean concisas o enciclopédicas, dado el carácter de la obra (vid. Prólogo, vi).

Terreros realiza una "proeza lexicográfica" ${ }^{21}$ de gran magnitud con esta obra única en el siglo XVIII, pues reúne todas las voces de artes y ciencias en un repertorio. Uno de los atributos destacables del Diccionario son los ejemplos y las referencias bibliográficas con los que autoriza las

20 El corpus del Diccionario lo completó Terreros con otras muchas fuentes textuales (Jacinto García 2008).

21 Tomo esta expresión de Pedro Álvarez de Miranda, cuando al referirse al Diccionario de Terreros escribe: “[...] proeza lexicográfica complementaria de la que unos años antes habían culminado los primeros académicos con la compilación del llamado Diccionario de Autoridades - pero con el mérito añadido, frente a este, de ser el de Terreros fruto de un esfuerzo individual" (Álvarez de Miranda 2008: 127). 
voces, sobre todo las más novedosas, garantizando así su empleo en la lengua real ${ }^{22}$. Para cada voz proporciona una información valiosa, clara y concisa, además de objetiva y veraz, dicha información está estrechamente relacionada con la salud pública y el bienestar, con un enfoque que hoy consideraríamos de carácter divulgativo; en cada artículo suele incluir también las equivalencias en francés, latín y algunas en italiano. Cuando lo estima conveniente, aporta la etimología, como leemos en: "antiartríticos, en la medicina, remedios contra la gota. Fr. anthiarthritiques; es voz griega de contra y gota". Y no duda en ampliar la definición si con ello contribuye a un mayor esclarecimiento: "antelmínticos, en la medicina los remedios contra lombrices, de los quales unos las sufocan y otros las deshacen. Fr. anthelminthiques, Lat. anthelminthica: los remedios antelmínticos son la coralina, la raíz de elecho, y todos los amargos; las reparaciones de mercurio; el aceite de almendras dulces; las nueces; avellanas y todas las semillas frías".

Terreros es consciente de que las voces de especialidad requieren una marca técnica que aplica mediante estructuras diversas, ya sea aludiendo directamente al ámbito temático como en: "lóbulo, término de Anatomía, lobo pequeño. Fr. Lobule. Lat. Lóbulus, cada lobo del pulmón, \&. está dividido en muchos lóbulos pequeños", "sarcoma, voz de medicina, excrescencia que proviene de la nutrición de la parte en que nace, sin concurso de otras. Fr. sarcoma, y vulgarmente Fil, o Fic de S. Fiacre" o "subsulto, voz médica, saltos continuados, v.g., en los tendones, V. Heister, trad. por Vázq.". Otras veces resuelve la inclusión de la marca técnica con una referencia a los profesionales que la emplean: "sonda, una especie de instrumento que introducen los cirujanos hasta encontrar la piedra si la hai en el que llaman mal de la orina, y sirve también para que esta salga. Fr. sonde. Lat. specillum"; o también: "alcoholar, entre los Químicos, destilar muchas veces algún licor o materia. Fr. Volatiser, rectifier. Lat. Destillatoriâ cucurbitâ elicere liquoris spiritus: así se saca el alcohol". Cuando la voz designa una actividad nueva, como en el neologismo ortopedia, sitúa el concepto en el vasto campo de las artes -con el significado que la voz artes poseía en el siglo ilustrado-: "ortopedia, arte de corregir las enfermedades y los defectos corporales en los niños". También tienen aquí su utilidad los verba dicendi, tan gratos a Terreros a lo largo de todo el Diccionario -cuando recurre a ellos para marcar las voces de oficios-: "sublingual, adj. que se dice en la anatomía de dos glándulas que están hacia el lado inferior de la lengua. Fr. sublingal. Lat. sub lingua situs", y también "luminosa, llaman en las Boticas al agua compuesta de piedra alumbre, agua rosada y llantén: sirve para quitar los granos de la cara y otros usos".

Otro rasgo distintivo en la microestructura del Diccionario de Terreros es la manera en que organiza las acepciones en cada lema. Cuando pertenecen al mismo ámbito temático las incluye dentro de un único artículo, como en: "alcohol, voz de Química y se toma por dos cosas mui distintas, lo primero por un polvo mui sutil y casi impalpable, y lo segundo por el espíritu de vino tan sutil a fuerza de destilaciones que no queda parte alguna flemosa", observamos que el orden establecido es el cronológico, de acuerdo con la vida de la palabra. Así mismo redacta otros artículos en los que las dos acepciones aparecen sucesivamente, sin apenas diferencia entre ambas, antes bien estableciendo la correlación que existe entre las dos voces: "meconio, término de Farmacia, jugo que se saca exprimiendo [...]. Fr. meconium. Por parecerse a este jugo, se da el mismo nombre al excremento negro y espeso, que se junta en las tripas del feto mientras dura el embarazo de la ma-

22 En este trabajo únicamente recogemos algunas de las entradas relacionadas con la medicina y áreas afines, con las que ejemplificamos algunos de los rasgos más destacados de la microestructura. 
dre; y que arroja o purga después de nacida la criatura. La voz se tomó del gr. $\mu \eta \kappa \omega v$ 'adormidera'. V. pez, y la obra de la crianz. fisic. de los niños, desde el destete, \&c. por Ballexerd, p.9 \&c."23. Sin embargo, cuando se trata de una voz polisémica con marcas técnicas diferentes las distribuye en artículos independientes, ordenando cada una de las entradas de acuerdo con la edad de cada una de las voces, así alveolo aparece en dos entradas, un primer artículo del ámbito de la botánica y otro con la voz de la medicina: "alveolo, en la Botánica la cavidad en que se contienen las semillas. Fr. Alveole. Lat. Alveolus. It. Alveolo", y a continuación "alveolo, en la medicina, las cavidades en que están arraigados los dientes y muelas. Fr. alveole, lat. alveolus, it. alveolo. Jeneralmente se puede decir alveolo entre sabios, hablando con naturalidad de qualquier especie de nicho o camita en que está colocada una semilla, insecto, feto, \&c.”.

Terreros maneja numerosas fuentes textuales: tratados clásicos y modernos, además de las traducciones más recientes, algunas coetáneas al repertorio que está componiendo. Como comprobamos al verificar las ediciones de algunas de las autoridades con que atestigua las voces, sobre todo cuando se trata de neologismos, por ejemplo, para autorizar la voz loquios, primero con la Nueva cartilla de matronas (1750) y a continuación con otra autoridad aún más reciente, la traducción de la obra del pediatra suizo Ballexerd, cuya primera edición fue publicada en 1765: "loquios, en las paridas, lo mismo que purgación. V. Y la nueva Cartilla de Matr., p. 69. Pero la Trad. de la obra de la crianza física de los niños de N. Ballejerd les llama Loquias. V. p.20 \&c.”; así mismo, la voz: "sebáceo, voz médica lo que pertenece a sebo o le contiene. Lat. sebaceus, vel sevaceus; así dicen humor sebáceo. V. los Princ. de Cir. de Mr. de la Faye, trad. por Xiorro”, también refrendada en fecha reciente, pues esta traducción de la obra de La Faye fue publicada en Madrid el año 1761.

Las fuentes lexicográficas constituyen el otro gran contingente del corpus, Jacinto García (2013: 285) nos recuerda que para el ámbito de especialidad de la medicina Terreros utiliza el Dictionnaire universel des drogues simples de Nicolas Lémery $(1759)^{24}$ y el Dictionnaire universel de médecine de James (1746-1748). Este último fue el mejor diccionario de medicina de su época, es muy probable que el jesuita tuviera los tres volúmenes de la versión francesa ${ }^{25}$, porque al definir algunos neologismos proporciona la misma información que da James en su diccionario, así podemos comprobarlo con la voz "amaurosis, pérdida de la vista sin aparecer la causa. V. Heister por Vázquez", que además autoriza con la traducción reciente, que el médico madrileño Santiago Vázquez había publicado en 1751, de la obra del gran iatromecánico Lorenz Heister. No es de extrañar, pues, que el léxico de la medicina que nos proporciona el Padre Terreros en su repertorio aporte la primera datación lexicográfica de numerosas voces neológicas, entre otras: necrosis, alveolo, amaurosis, aponeurótico, ascítico, atrófico, sebáceo, loquios, etc.

23 Este artículo muestra cómo Terreros introduce el neologismo semántico meconio tomado de la reciente traducción que había hecho Eugenio de Llaguno del tratado de pediatría del médico suizo Ballexerd.

24 El diccionario de Nicolas Lèmery (1645-1715) alcanzó gran notoriedad en toda Europa. Fue publicado por primera vez en París en 1698 y se mantuvo de actualidad durante más de un siglo gracias a las numerosas ediciones aparecidas en París, Amsterdam y Roterdam los años 1714, 1715, 1716, 1723, 1726, 1727, 1733, 1738, 1748, 1759, 1760 y 1807, además de una versión en italiano publicada en Venecia en 1737 (Lafont 2009: 273). Dado el flujo de libros que existía en la Europa dieciochesca, pensamos que Terreros pudo disponer en su celda del Seminario de Nobles de cualquiera de las numerosas ediciones que vieron la luz en la primera mitad de la centuria.

25 El Dictionnaire universel de médecine había sido traducido por Diderot, Eidous y Toussaint desde el original que R. James había publicado en lengua inglesa entre los años 1743-1745, y fue editado en París entre 1746 y 1748. 


\section{Conclusión}

La lexicografía de especialidad en lengua española alcanza su madurez en el siglo ilustrado, la renovación de la ciencia favorece el nacimiento de las nuevas terminologías, con el consecuente florecimiento de los vocabularios de especialidad. Es en este contexto donde las terminologías comenzarán su andadura, primero con el desarrollo de los llamados glosarios escondidos y posteriormente con la creación de los repertorios de especialidad.

\section{Referencias bibliográficas}

Álvarez de Miranda, P. (2008). Perfil biográfico del Padre Terreros. In S. Larrazábal, \& C. Gallastegui (Eds.), Esteban de Terreros y Pando: vizcaíno, polígrafo y jesuita. III Centenario: 1707-2007 (pp. 127-142). Bilbao: Universidad de Deusto. . (2011). Los diccionarios del español moderno. Gijón: Ediciones Trea.

Astigarraga, J.; \& Zabalza, J. (2006). La economía en los diccionarios y las enciclopedias del siglo XVIII en España. Documentos de trabajo de la Asociación Española de Historia Económica, 607. <https://www.aehe. es/wp-content/uploads/2015/04/dt-aehe-06071.pdf>

Bahí, J. F. [Plenck, J. J.] (1802). Elementos de la nomenclatura botánica y sistema sexual de las plantas. Por Joseph Jacobo Plenk, traducido del latín al español. Barcelona: Compañía de Jordi, Roca y Gaspar.

Capel, H. (1981). Los diccionarios geográficos de la Ilustración española. Cuadernos críticos de Geografía Humana, VI-31. <http://www.ub.edu/geocrit/geo31.htm>

Colmeiro, M. (1858). La botánica y los botánicos de la Península Hispano-Lusitana: estudios bibliográficos y biográficos. Madrid: Imprenta y Estereotipia de M. Rivadeneira.

Cordón García, J. A. (1996). La constitución del enciclopedismo moderno de la Ilustración al Hipertexto. Boletín de la ANABAD, 46, 2, 113-128.

Galisteo Xiorro, J. [La Faye, G.] (1761). Principios de cirugía traducidos del francés por D. Juan Galisteo Xiorro. Madrid: A. Marín.

Gómez de Enterría, J. (1996). Voces de la economía y el comercio en el español del siglo XVIII. Alcalá de Henares: Servicio de Publicaciones de la Universidad de Alcalá.

. (2008). El Padre Terreros traductor de la obra de Pluche. In S. Larrazábal, \& C. Gallastegui (Eds.),

Esteban de Terreros y Pando: vizcaíno, polígrafo y jesuita. III Centenario: 1707-2007 (pp. 249-273). Bilbao: Universidad de Deusto.

. (2012). El vocabulario médico de los novatores en el siglo XVIII. In M. T. García Godoy (Ed.), El español del siglo XVIII. Cambios diacrónicos en el primer español moderno (pp. 55-81). Berna: Peter Lang.

- (2020). El vocabulario de la medicina en el español del siglo XVIII. Berna: Peter Lang.

Gutiérrez Rodilla, B. (2010). Sobre lexicografía médica del renacimiento castellano: los vocabularios de Andrés Laguna y Bartolomé Hidalgo de Agüero. Revista de Lexicografía, 16, 59-74.

Jacinto García, E. J. (2008). Terminología y autoridades científico-técnicas en el Diccionario Castellano (1786-1793) del P. Terreros. In S. Larrazábal, \& C. Gallastegui (Eds.), Esteban de Terreros y Pando: vizcaíno, polígrafo y jesuita. III Centenario: 1707-2007 (pp. 765-794). Bilbao: Universidad de Deusto. 
. (2013). El principio de autoridad en los diccionarios generales del español (siglos XVIII-XX) (Tesis doctoral inédita). Madrid: Universidad Complutense de Madrid. <https://eprints.ucm.es/20660/1/ T34377.pdf>

James, R. (1746-1748). Dictionnaire universel de médecine, traduit de l'anglais par Mrs. Diderot, Eidous et Toussaint. Paris: Briasson, David l'Aîné, Durand.

Lafont, O. (2009). Nicolas Lémery, providence des bibliophiles. Revue d'histoire de la pharmacie, 96, 363, 267-276. <http://www.persee.fr/doc/pharm_0035-2349_2009_num_96_363_22073>

Linneo [Linnaeus], K. von (1736). Fundamenta Botanica. Amsterdam.

Llaguno y Amírola, E. [Ballexerd, J.] (1765 [1787]). Crianza física de los niños desde su nacimiento hasta la pubertad, y método el más seguro de robustecer la especie humana contra los insultos de las enfermedades: disertación premiada por la Sociedad Holandesa de las ciencias en 1762 y trad. por D. Patricio de España. Madrid: Antonio Espinosa.

Real Academia Española (1726, 1734, 1737, 1739): Diccionario de la lengua castellana, en que se explica el verdadero sentido de las voces, su naturaleza y calidad, con las phrases o modos de hablar, los proverbios o refranes, y otras cosas convenientes al uso de la lengua [...]. Compuesto por la Real Academia Española. Madrid: Imprenta de Francisco del Hierro.

Santamaría, F. J. de (1942). Diccionario general de Americanismos. Méjico D.F.: Editorial Pedro Robredo.

Suárez de Ribera, F. (1730). Clave medico-chirurgica universal y Diccionario medico, chyrurgico, anathomico, mineralogico, botanico, zoologico, pharmaceutico, chymico, historico-phisico. Madrid: Imprenta de la Viuda de Francisco del Hierro.

. [Curvo Semedo, J.] (1733). Observaciones de Curvo compendiadas e ilustradas con admirables arcanos medicinales. Madrid: Imprenta de Domingo Fernández de Arrojo.

Terreros y Pando, E. de [Pluche, M.] (1753-1755). Espectáculo de la Naturaleza, o Consideraciones a cerca de las particularidades de la Historia Natural que han parecido más a propósito para excitar una curiosidad útil y formarles la razón a los Jóvenes Lectores, por el Abad M. Pluche, y traducido al castellano. Madrid: Gabriel Ramirez.

. (1767) [1786-1788]. Diccionario castellano con las voces de ciencias y artes y sus correspondientes en las tres lenguas francesa, latina e italiana [...]. Madrid: Viuda de Ibarra.

Vidal, D. [Plenck, J. J.] (1797). Tratado de las enfermedades de los ojos de Josef Jayme Plenck [...]. Traducido del latín por el Dr. D. Domingo Vidal. Cádiz: Imprenta de D. Manuel Ximénez Carreño.

West, C. B. (1932). La théorie de la traduction au XVIIIe siècle par rapport surtout aux traductions françaises d’ouvrages anglais. Revue de Littérature Comparée, 12, 330-355.

Zabía Lasala, M. P. (ed.) (1999). Diccionario de Juan Alonso y de los Ruyzes de Fontecha, 1606. Madrid: Arco Libros. 
